index ocomunicación | no 10(3) 2020 | Páginas 235-261

E-ISSN: 2174-1859 | ISSN: 2444-3239 | Depósito Legal: M-19965-2015

Recibido el 29_10_2020 | Aceptado el 31_10_2020 | Publicado el 12_11_2020

\title{
USO DE SCOPUS Y WEB OF SCIENCE PARA INVESTIGAR Y EVALUAR EN COMUNICACIÓN SOCIAL: ANÁLISIS COMPARATIVO Y CARACTERIZACIÓN
}

\author{
USE OF SCOPUS AND WEB OF SCIENCE TO RESEARCH \\ AND EVALUATE IN SOCIAL COMMUNICATION: \\ COMPARATIVE ANALYSIS AND CHARACTERIZATION
}

https://doi.org/10.33732/ixc/10/03Usodes

\author{
Lluís Codina \\ Universitat Pompeu Fabra \\ lluis.codina@upf.edu \\ http://orcid.org/0000-0001-7020-1631
}

\begin{abstract}
Alejandro Morales-Vargas Universidad de Chile | Universitat Pompeu Fabra amorales@uchile.cl http://orcid.org/0000-0002-5681-8683
\end{abstract}

\section{Ruth Rodríguez-Martínez \\ Universitat Pompeu Fabra ruth.rodriguez@upf.edu http://orcid.org/0000-0001-5633-6126}

\section{Mario Pérez-Montoro}

Universitat de Barcelona perez-montoro@ub.edu http://orcid.org/0000-0003-2426-8119

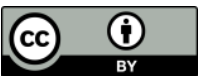

Para citar este trabajo: Codina, L., Morales-Vargas, A., RodríguezMartínez, R. y Pérez-Montoro, M. (2020). Uso de Scopus y Web of Science para investigar y evaluar en comunicación social: análisis comparativo y caracterización. index.comunicación, 10(3), 235-261.

https://doi.org/10.33732/ixc/10/03Usodes 


\section{Este trabajo forma parte del proyecto «Narración interactiva y visibilidad digital en el documental interactivo y el periodismo estructurado». RTI2018-095714-B-C21 (MICINN/FEDER), Ministerio de Ciencia, Innovación y Universidades (España)}

Resumen: El objetivo es caracterizar y comparar las opciones que ofrecen las principales bases de datos a los investigadores en comunicación social para buscar y evaluar información académica. Para ello, mediante una evaluación experta se examinaron las características funcionales de Scopus y Web of Science. Como resultado se presenta una revisión detallada de dimensiones como la cobertura, la recuperación de información y las herramientas de análisis de fuentes y autores presentes en cada una, además de métricas de impacto específicas. Entre las conclusiones, se observan similitudes en las funciones disponibles, pero diferencias significativas en el número de revistas en ámbito de las ciencias sociales y las humanidades, lo que deja a Scopus en mejor posición en el caso de tener que optar.

Palabras clave: Scopus; Web of Science; comunicación social; ciencias sociales; humanidades; investigación.

Abstract: The objective is to characterize and compare the options offered by the main databases to researchers in social communication to search and evaluate academic information. To do this, through an expert evaluation, the functional characteristics of Scopus and Web of Science were examined. As a result, a detailed review of dimensions such as coverage, information retrieval and analysis tools of sources and authors present in each one is presented, as well as specific impact metrics. Among the conclusions, similarities are observed in the available functions, but significant differences in the number of journals in the field of social sciences and humanities, which leaves Scopus in a better position in the case of having to choose.

Keywords: Scopus; Web of Science; Social Communication; Social Sciences; Humanities; Research. 


\section{Introducción}

Como es sabido, una base de datos académica es un sistema de información que proporciona funciones de acceso a la información y de análisis de documentos publicados o bien por editoriales científico-académicas o bien por otros agentes del mundo académico, tales como asociaciones científicas o universidades.

Estos documentos pueden responder a una amplia tipología, pero en general corresponden a la siguiente:

1. Artículos publicados en revistas científicas, esto es, que se guían por el procedimiento denominado peer review.

2. Comunicaciones procedentes de actas de congresos de carácter académico.

3. Capítulos de libros de editoriales, también de tipo académico.

Eventualmente, algunas bases de datos de este tipo pueden incluir aún otras tipologías, como patentes o ensayos clínicos. No obstante, los tres tipos de documentos señalados constituyen el grueso de las bases de datos académicas en casi todas las disciplinas y, en especial, en el ámbito de las ciencias humanas y sociales, que es el que nos interesa aquí. De estos tres, los artículos de revistas académico-científicas son, con diferencia, los principales.

En este contexto, la oportunidad de analizar, ya sea de forma unitaria o como parte de un análisis comparativo, una base de datos académica puede tener diversas motivaciones y objetivos. En algunos escenarios, los responsables de una biblioteca universitaria o de un centro de investigación necesitarán valorar posibles suscripciones de revistas o recomendaciones para sus usuarios.

En otro, tal vez sea un estudioso del diseño de interfaces o del funcionamiento de los sistemas de información quien necesite elementos de apoyo en sus análisis. En otro, el contexto tal vez sea simplemente - o nada menos- la necesidad de un formador de poder disponer de elementos incisivos de análisis con los que preparar sus estrategias de docencia. Por último, puede ser un equipo investigador el que necesite estudiar y comparar bases de datos para decidir sobre la utilización en su proyecto.

\subsection{Objetivos}

Como sea, en este trabajo nos proponemos un doble objetivo: por un lado, presentar las dimensiones principales de un análisis de bases de datos académicas, en general; $y$, por otro, aplicarlos al caso concreto de la investigación en comunicación social a través de la comparación entre las bases de datos Scopus y Web of Science (WoS). 


\subsection{Dimensiones de análisis}

Gracias a otros trabajos (Codina, 2011, 2017, 2018) que, a su vez, están en línea con la corriente principal en este terreno de análisis (Gasparyan, 2013), podemos establecer que tres de las principales dimensiones de análisis de una base de datos son las siguientes:

a) La cobertura, esto es, las dimensiones cuantitativas y cualitativas de sus colecciones, así como el grado de solapamiento relativo en caso de comparaciones entre diferentes bases de datos.

b) Las funciones de recuperación de información, lo que incluye la búsqueda, la navegación, el descubrimiento y la explotación de la información.

c) Las funciones de análisis de la información, esto es, el tipo de métricas y las facilidades de análisis que cada base de datos proporciona.

\subsection{Justificación}

Como ya hemos señalado, el objeto de estudio al que aplicaremos las tres dimensiones anteriores son las dos bases de datos sobre las que existe un mayor consenso internacional acerca de su decisiva importancia académica, a saber, las bases de datos Scopus y WoS (Leydesdorff et al., 2016). Esta importancia deriva, al menos, de tres aspectos:

Primero, debido a la calidad intrínseca de ambas, ya que son dos bases de datos multidisciplinares que cubren un gran número de revistas y -al menos en principio- son las que llevan a cabo una selección más rigurosa de las publicaciones que pueden formar parte de la misma (Kousha et al., 2010). De este modo, en teoría, formar parte de una de estas bases de datos se considera un mérito de la revista en cuestión.

Segundo, por su importancia estratégica, ya que, en función de lo indicado en el punto anterior, son las dos bases de datos que se utilizan de forma mayoritaria en procesos de análisis y evaluación de la ciencia.

Tercero, porque se da la circunstancia de que son las dos bases de datos académicas que aportan un mejor apartado de análisis de la información académica, a través de sus métricas, índices y funcionalidades para tales efectos.

En lo que sigue, procederemos a presentar una comparativa de las dos bases de datos en función de las dimensiones señaladas, haciendo énfasis en la temática de la comunicación social (Chung \& Park, 2012; Costa-Sánchez, 2017; Demeter, 2018b, 2018a; Túñez-López, 2013). La razón de la elección de este tema es debido a su gran especificidad dentro de la amplia gama de estudios en ciencias sociales y humanidades (Delgado-López-Cózar \& Repiso-Caballero, 
2013). Elegir una especialidad así, altamente específica, nos ayuda a caracterizar las dos bases de datos que son objeto de este trabajo.

\section{Metodología}

Para cumplir con el doble objetivo planteado, en primer lugar se aplicó el método del análisis experto mediante evaluación heurística (Morales-Vargas et al., 2020), inspeccionando en detalle cada uno de los componentes de las bases de datos académicas y describiendo las funcionalidades que sus respectivas interfaces brindan a los usuarios (Weinhold et al., 2011), tanto para la búsqueda de información como para el análisis de impacto.

Luego, se realizó una comparación entre las bases de datos Scopus y Web of Science, revisando la cobertura que presenta cada una específicamente para la disciplina de la comunicación social (de-Filippo, 2013; Rogel-Salazar et al., 2017) y detectando intersecciones en los títulos de revistas.

En el caso de las fuentes, utilizamos las listas oficiales de cada una de las dos bases de datos, para lo cual hicimos un mapeo científico del número de revistas respectivas en comunicación social (Montero-Díaz et al., 2018) en cada una, así como de su grado de solapamiento. Para el caso de las ecuaciones, llevamos a cabo una serie de búsquedas que nos ayudaron a complementar nuestros análisis de cobertura.

Las fuentes respectivas que hemos consultado son las siguientes:

1. Scopus: https://www.scopus.com/sources.

2. WoS: https://mjl.clarivate.com/search-results y https://clarivate.libguides.com/webofscienceplatform/coverage.

En los dos casos, los enlaces señalados nos llevan a los sitios en los que podemos consultar las listas respectivas de publicaciones que forman parte de cada una de las bases de datos. Los datos que mostraremos a continuación corresponden al mes de octubre de 2020.

\section{Resultados}

A continuación, se presentan los principales hallazgos del estudio según las dimensiones identificadas en las bases de datos académicas: cobertura, recuperación de información y análisis de la información, estableciendo una comparativa entre Scopus y WoS.

\subsection{Cobertura}

Las dos principales bases de datos usadas por la comunidad científica internacional presentan una vasta cobertura en todas las áreas del conocimiento. No 
obstante, la tabla 1 evidencia que Scopus presenta un número total significativamente mayor de revistas en su colección.

Tabla 1. Total de revistas en todas las disciplinas

\begin{tabular}{|l|r|}
\hline \multicolumn{1}{|c|}{ Base de datos } & Total de revistas en todas las disciplinas \\
\hline Scopus & 38.589 \\
\hline Web of Science & 24.775 \\
\hline
\end{tabular}

Fuente: Scopus y WoS (octubre 2020).

Lo propio ocurre al analizar el caso específico del total de revistas en ciencias sociales y humanidades (tabla 2), donde observamos que esta relación se mantiene y es Scopus la base de datos que contiene más títulos.

Tabla 2. Revistas en ciencias sociales y humanidades

\begin{tabular}{|l|r|}
\hline \multicolumn{1}{|c|}{ Base de datos } & Revistas en cs. sociales y humanidades \\
\hline Scopus & 9.451 \\
\hline Web of Science & 6.500 (estimación) \\
\hline
\end{tabular}

Fuente: Scopus y WoS (octubre 2020).

Cabe señalar que todos los datos relativos a WoS son más difíciles de establecer si vamos más allá del número total de revistas de su colección principal, la denominada Core Collection, que es la referencia que hemos tomado para WoS en la tabla 1. Para la tabla 2, en cambio, establecer el número total de revistas en ciencias sociales y humanidades es fácil en el caso de Scopus.

Para WoS debemos hacer estimaciones suponiendo una proporción similar de revistas de ciencias sociales y humanidades en relación al total de revistas en su índice Emerging Sources Citation Index (ESCI) que en los otros de la colección principal, ya que los datos en ESCI no están desagregados por áreas temáticas. En todo caso, la extrapolación de tales proporciones relativas sugiere la cifra indicada en la tabla 2 para el total de revistas de ciencias sociales y humanidades en WoS (incluyendo ESCI, es decir, el total de revistas de la colección principal).

Tabla 3. Revistas en la categoría comunicación social

\begin{tabular}{|l|r|}
\hline \multicolumn{1}{|c|}{ Base de datos } & Revistas en comunicación social \\
\hline Scopus & 445 \\
\hline Web of Science & 300 \\
\hline
\end{tabular}

Fuente: Scopus y WoS (octubre 2020). 
A su vez, en la tabla 3 consideramos el número total de revistas bajo la categoría específica de comunicación social en las dos bases de datos.

Este cálculo, de nuevo, es sumamente fácil en Scopus, pero también lo es en este caso en WoS gracias a la aplicación denominada Master Journal List de Clarivate.

Todo lo anterior no significa que los títulos de Scopus sean un superconjunto de los títulos de WoS. Es decir, no podemos afirmar que consultando Scopus tengamos acceso a los mismos títulos de WoS y, además, otros títulos. Consultando Scopus accedemos a la mayor parte de los títulos que también están en WoS, pero no a todos.

Tampoco podemos estar seguros de la exactitud de la ratio indicada, porque la categorización de revistas no es homogénea. De este modo, en realidad solamente podemos estar seguros de que Scopus tiende a tener mayor cobertura relativa que WoS, pero no podemos saber si corresponde a la ratio que aparece utilizando estos resultados.

En el caso específico de la disciplina de la comunicación, se constata un alto número de revistas que están indexadas en ambas bases de datos, según muestra la imagen 1, generada a partir del análisis con la herramienta SCImago Journal Rank. Aún con ese nivel de solapamiento, Scopus presenta 200 títulos que no están presentes también en WoS.

\section{Imagen 1. Solapamiento en revistas de comunicación}

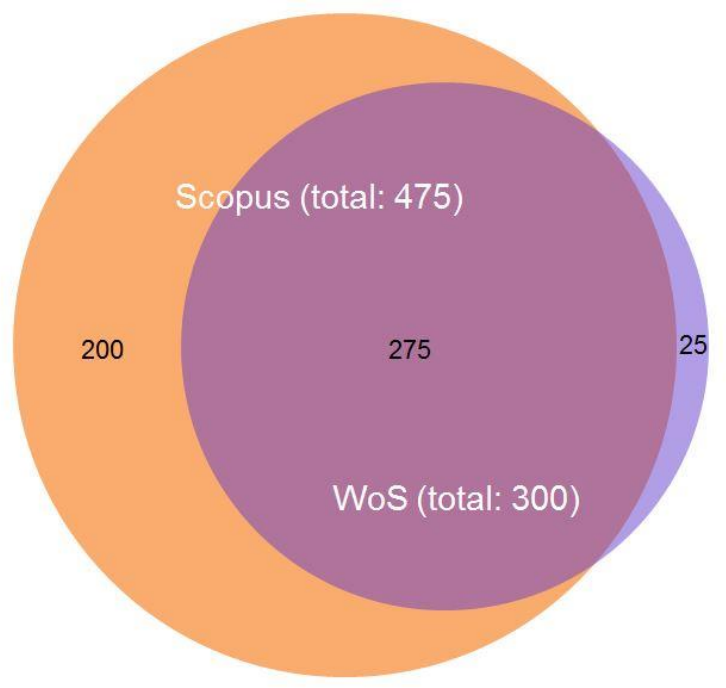

Fuente: Elaboración propia, con datos de SCImago y WoS (octubre 2020). 


\subsection{Recuperación de información}

En los últimos años, se ha producido una convergencia de diseño funcional entre estas dos bases de datos. El resultado es que ambas adoptan los mismos patrones (patterns), pero cada una con una adaptación específica.

Como parte del análisis, se muestran las posibilidades de búsqueda avanzada de cada una de las bases de datos, aunque es posible adelantar una alta similitud funcional gracias a estudios anteriores.

La búsqueda avanzada de ambas bases de datos se beneficia del uso de un patrón de enorme eficacia, al que denominamos pattern de filas apiladas.

Su principal característica es que permite expresar por igual búsquedas simples o de una alta complejidad, gracias a un diseño basado en filas que representan conceptos, y la posible articulación de dos o más filas mediante operadores de búsqueda. En lo que sigue, presentamos capturas de cada base de datos.

\subsubsection{Búsqueda avanzada en Scopus}

El punto principal de fortaleza de este formulario es que permite añadir o eliminar filas, siendo que cada fila puede representar un concepto distinto de la necesidad de información.

Además, las filas pueden relacionarse entre ellas mediante operadores booleanos, tal como vemos en la imagen 2 . Entre las dos filas podemos ver un menú desplegable con el operador $A N D$ preseleccionado, aunque al desplegar el menú se puede optar también por el $O R$ y el NOT.

Imagen 2. Formulario de búsqueda avanzada de Scopus



Fuente: captura de pantalla de Scopus.

Por último, cada fila puede estar asignada a un grupo de campos, como vemos en la imagen 3 (grupo temático por defecto). Esta combinación de prestaciones permite búsquedas muy precisas. 
Imagen 3. Ejemplo de búsqueda avanzada en Scopus, combinando dos conceptos y con limitación de fechas de publicación

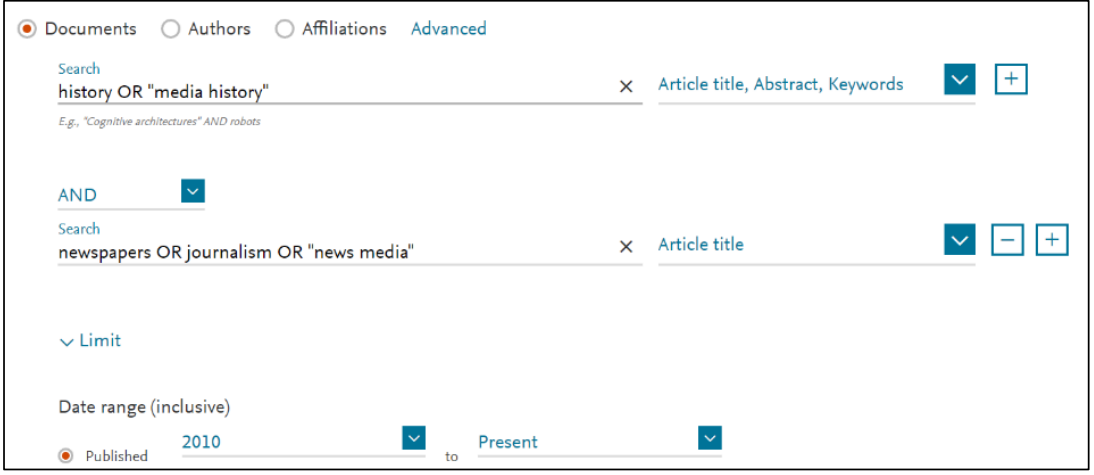

Fuente: captura de pantalla de Scopus.

En la captura precedente podemos ver un ejemplo significativo de uso de la búsqueda avanzada que nos permite ilustrar el potencial que este modelo de formulario permite a los investigadores:

1. Hemos combinado los conceptos [historia] y [periodismo] mediante el grupo de palabras clave: history, media history, por un lado, y newspapers, journalism, news media, por otro.

2. Cada grupo de sinónimos lo hemos relacionado con un $O R$ entre ellos, y los dos conceptos los hemos intersectado con un AND.

3. Finalmente, hemos limitado la búsqueda a artículos publicados a partir del 2010.

Desde el punto de lógico, corresponde a la siguiente ecuación de búsqueda booleana (la restricción a los campos respectivos no la representamos aquí, ni tampoco la restricción por rango de años, a efectos de mayor claridad): [history OR "media history"] AND [newspapers OR journalism OR "news media"].

\subsubsection{Búsqueda avanzada en Web of Science}

Como veremos por la siguiente captura de pantalla de Web of Science (imagen 4), proporciona un formulario de búsqueda avanzada basado en el mismo pattern.

En este caso, mostramos su utilización con la misma necesidad de información: 
Imagen 4. Formulario de búsqueda avanzada de WoS

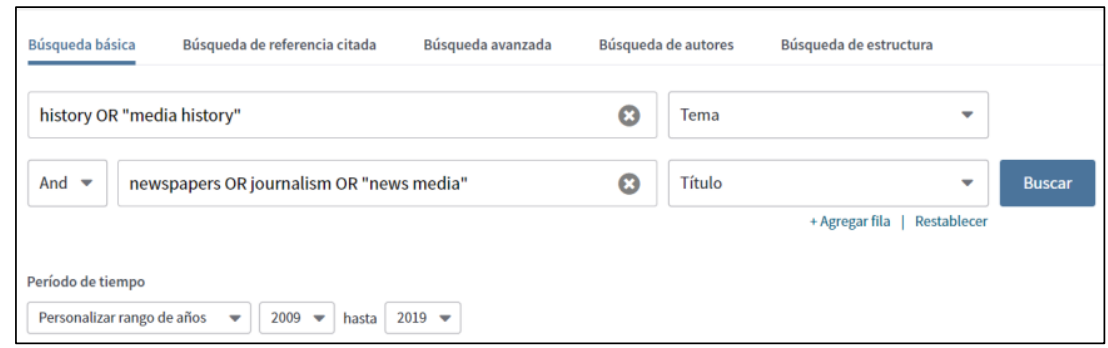

Fuente: captura de pantalla de WoS.

De esta manera, el diseño del formulario de la base de datos WoS nos ha permitido expresar exactamente la misma ecuación con la que hemos puesto a prueba la otra base de datos.

En los dos casos, hemos podido representar sin ningún problema la misma ecuación de búsqueda booleana: [history OR "media history"] AND [newspapers OR journalism OR "news media"].

En los dos casos, además, la búsqueda anterior se pudo parametrizar, como mostramos a continuación gracias al uso de los respectivos formularios de búsqueda:

1. [history OR "media history"] = campos temáticos más título (título, resumen, palabras clave).

2. [newspapers OR journalism OR "news media" ] = campo título.

Para esta dimensión de análisis, podemos concluir que las dos bases de datos ofrecen funcionalidades ampliamente equivalentes. Ambas presentan un excelente sistema de recuperación de información, gracias a la utilización de formularios de búsqueda avanzada bien diseñados. En los dos casos, las páginas de resultados resultantes ofrecen, además, prestaciones de ordenación y de exportación exhaustivas.

En este apartado, por consiguiente, tanto Scopus como Web of Science aportan el mismo valor para los investigadores. Apenas pueden detectarse diferencias de diseño o diferencias estéticas pero, en este caso, tales diferencias escapan a los aspectos que podemos valorar aquí.

\subsection{Análisis de la información}

Estos análisis se encuentran en diferentes apartados de las bases de datos, pero son de dos tipos distintos: de características de los documentos y de características de las citaciones recibidas por los mismos. 
Los análisis de las características de los documentos proporcionan distribuciones de los mismos por diferentes facetas: año de publicación, fuentes - revistas, editoriales o congresos-, autores, áreas temáticas, países, etc.

Los análisis de las características de las citaciones proporcionan datos detallados de las citas que reciben los documentos encontrados: total de citas recibidas, índice $h$, citas por años, citas por documento, entre otros, así como las referencias citantes, lo que siempre añade factores de descubrimiento.

Las herramientas correspondientes tienen en común que requieren, o son consecuencia de, una operación de búsqueda previa, y están disponibles en tres apartados distintos:

a) En los filtros de la página de resultados, normalmente ubicados en lateral izquierdo de la misma, según el pattern habitual.

b) En una página ad hoc de análisis de documentos, que se genera al activar un enlace en la parte superior de la página de resultados.

c) En una página ad hoc de análisis de citaciones que se genera, al igual que en la anterior, al activar un enlace disponible en la página de resultados.

\subsubsection{Análisis de la información en Scopus}

En el caso de Scopus, a partir de una búsqueda, vamos a poder analizar el conjunto de documentos resultantes en estos tres apartados:

a) Filtros de la página de resultados.

La captura de pantalla siguiente (imagen 5), generada a partir de un ejemplo diferente al de la dimensión de análisis anterior, muestra el resultado de la ecuación de búsqueda y los tres puntos que nos van a proporcionar análisis de estos.

Al mismo tiempo, la misma imagen permite apreciar, en la barra de navegación lateral izquierda, los filtros de la página de resultados de búsqueda, que brinda la posibilidad de refinarlos por diversos criterios o facetas, tales como: tipo de acceso, año de publicación, nombre de autor, disciplina científica, estado de publicación, tipo de documento, título de la fuente, palabra clave, afiliación, auspiciador, país, tipo de fuente e idioma. Estos filtros se pueden utilizar como criterio de inclusión o exclusión, y aplicar de manera combinada. 




Fuente: captura de pantalla de Scopus.

b) Análisis de los documentos.

Además de los filtros, tenemos la posibilidad de acceder a una página ad hoc donde la base de datos muestra un análisis de la página de resultados mediante distribuciones por diferentes facetas.

Algunas de estas, coinciden con las que se muestran en los filtros - que ya hemos señalado-, pero en esta sección específica se muestran con mayor riqueza informativa y con apoyo de recursos gráficos.

El acceso a esta página, en el caso de Scopus, se presenta como un enlace rotulado Analyze search results (imagen 5).

Al hacer clic en Analyze search results, cambiamos la página de resultados por una nueva página (imagen 6) donde podemos explorar las características de los documentos encontrados con base en siete distribuciones distintas: documentos por año, por fuentes (revistas), autor, afiliación (lugar de trabajo), países, tipo de documento y áreas temáticas. 
Imagen 6. Ejemplo de distribuciones de datos en Scopus

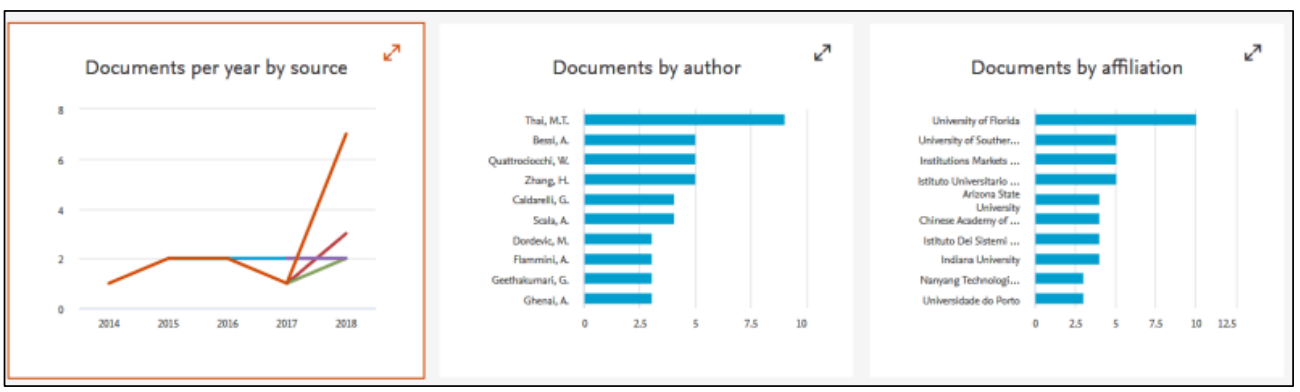

Fuente: captura de pantalla de Scopus.

Otras distribuciones corresponden a años y fuente, autor y afiliación o lugar de trabajo. Como es posible apreciar, cada uno de los cuadros mostrados en la captura precedente se puede ampliar, en cuyo caso muestran información más detallada y en un formato doble, como veremos en la imagen siguiente (imagen 7).

Imagen 7. Ejemplo de una distribución de datos ampliada en Scopus

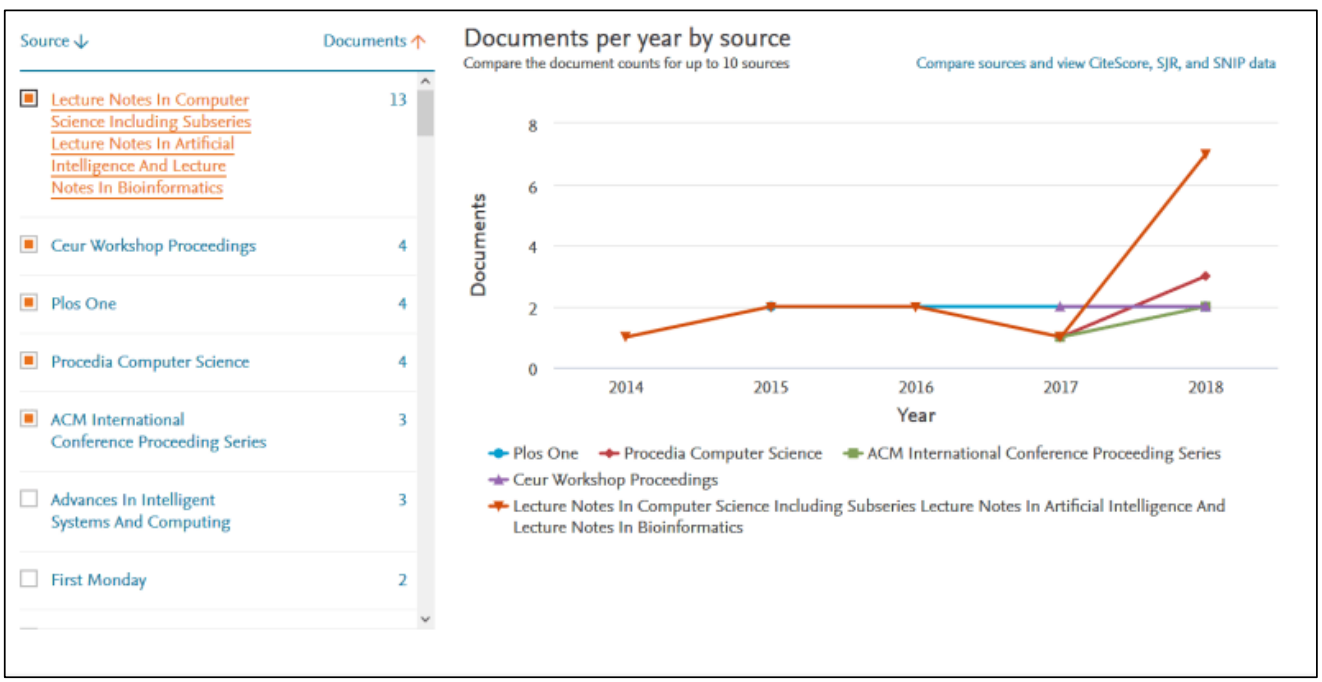

Fuente: captura de pantalla de Scopus.

Finalmente, cabe señalar que existen más tipos de distribuciones en Scopus, como es el caso de la información por países, tipos de documentos y área temática, tal como se presenta en la imagen 8. 
Imagen 8. Otro ejemplo de distribuciones de Scopus: países, tipos de documentos y área temática



Fuente: captura de pantalla de Scopus.

c) Análisis de citaciones.

El análisis de citaciones, indicado con un enlace rotulado View citation overview en el caso de Scopus, está disponible tras marcar dos o más referencias. Esto permite ver los datos una parte de las mismas, o de todas las referencias si tal cosa tiene sentido para nosotros.

Al acceder a la opción Citation overview (imagen 9), obtenemos como primera información un resumen gráfico de las citas recibidas por los documentos encontrados como respuesta a la consulta.

\section{Imagen 9. Distribución de citas por años del conjunto de referencias} seleccionadas



Fuente: captura de pantalla de Scopus. 
Imagen 10. Lista de documentos con resultados del análisis de citas, ordenadas de mayor a menor número de citas recibidas

\begin{tabular}{|c|c|c|c|c|c|c|c|c|c|c|c|}
\hline & Documents & Citations & $<2015$ & 2015 & 2016 & 2017 & 2018 & 2019 & Subtotal & $>2019$ & Total \\
\hline & & Total & 34 & 67 & 160 & 233 & 470 & 17 & 947 & 0 & 981 \\
\hline$\square 1$ & The spreading of misinformation online & 2016 & & & 30 & 43 & 75 & 3 & 151 & & 151 \\
\hline$\square 2$ & Science vs conspiracy: Collective narratives in the age of $\mathrm{m} . .$. & 2015 & & 7 & 13 & 20 & 36 & & 76 & & 76 \\
\hline$\square 3$ & Containment of misinformation spread in online social networ... & 2012 & 12 & 7 & 13 & 16 & 10 & 2 & 48 & & 60 \\
\hline$\square 4$ & Ebola, Twitter, and misinformation: A dangerous combination? & 2014 & 1 & 16 & 14 & 8 & 14 & 2 & 54 & & 55 \\
\hline$\square 5$ & With Facebook, blogs, and fake news, teens reject journalist... & 2012 & 1 & 5 & 3 & 14 & 30 & & 52 & & 53 \\
\hline$\square 6$ & Detecting misinformation in online social networks using cog... & 2014 & & 5 & 9 & 10 & 20 & 2 & 46 & & 46 \\
\hline$\square 7$ & Misinformation propagation in the age of Twitter & 2014 & & 3 & 10 & 10 & 16 & 2 & 41 & & 41 \\
\hline$\square 8$ & Story and science: How providers and parents can utilize sto... & 2013 & 3 & 8 & 10 & 8 & 12 & & 38 & & 41 \\
\hline$\square 9$ & A social diffusion model of misinformation and disinformatio... & 2013 & 8 & 4 & 7 & 5 & 7 & & 23 & & 31 \\
\hline$\square 10$ & Fake News: The narrative battle over the Ukrainian conflict & 2016 & & & & 9 & 19 & 1 & 29 & & 29 \\
\hline
\end{tabular}

Fuente: captura de pantalla de Scopus.

En la captura precedente, tenemos el gráfico de la distribución del número de citas de las referencias de la página de resultados. Al igual que otros gráficos, es interactivo. Al situar el cursor en un punto, aparece una ficha con el número de cita (vemos que, por ejemplo, en el año 2015 fueron 67) y un enlace a las referencias citantes.

La misma información de citaciones anterior, en este caso en forma de tabla (imagen 10) que puede ser ordenada con diversos criterios y con datos detallados por años.

En este formato tenemos más información, como el total de citas recibidas por el conjunto de los artículos, así como los datos año a año. También tenemos la posibilidad de acceder a los artículos de los que proceden las citas, ya que todas y cada una de las cifras de datos son enlaces a los artículos de los que proceden las citas.

También podemos hacer clic en el número acumulado de citas (en la parte superior derecha de la tabla), con lo cual accedemos a una página de resultados con la totalidad de los artículos citantes. 


\section{Imagen 11. Índice $h$ del conjunto de resultados mediante un gráfico interactivo}



Fuente: captura de pantalla de Scopus.

Disponemos, por último, de un recuadro (imagen 11) donde se muestra de forma gráfica el índice $h$. Vemos en el gráfico el punto exacto donde el número de citaciones (eje $y$ ) y el número de documentos (eje $x$ ) coincide. El cruce señala el índice $h, 17$ en este caso, indicando que 17 documentos tienen al menos 17 citas.

\subsubsection{Análisis de información en Web of Science}

Las opciones de análisis de la página de resultados, en el caso de Web of Science, son equivalentes a Scopus, con un solapamiento lógico en los indicadores más significativos, pero con algunas diferencias en el número de opciones concretas disponibles - WoS presenta algunas más- y en la forma de presentación de los datos.

Las ubicaciones también son las mismas, a saber: filtros disponibles en el lateral izquierdo de la página de resultados (imagen 12), que actúan como una minería de datos; una página ad hoc de análisis de documentos, y una página de análisis de citaciones.

a) Filtros de la página de resultados.

Una de las diferencias es que en WoS no es necesario marcar resultados para obtener un análisis de citaciones. Esto simplifica las operaciones pero limita las opciones. A cambio, WoS presenta alguna opción de análisis adicional e incluye un formato de visualización muy atractivo: los mapas de árbol. En conjunto, toda la página de análisis de resultados es especialmente funcional y de fácil y agradable diseño. 


\section{Imagen 12. Puntos de acceso a los análisis en la página de resultados de WoS}



Fuente: captura de pantalla de WoS.

En la cabecera de la página de resultados (imagen 13), tras una consulta, tenemos las dos opciones de análisis en la parte superior derecha: Analizar resultados (documentos) y Crear informe de citas.

Imagen 13. Ubicación del enlace a la página de análisis en la cabecera de la página de resultados de WoS

\begin{tabular}{|llll}
\hline Ordenar por: Fecha & Veces citado Conteo de uso Relevancia \\
$\square$ Seleccionar página & $\square 5 K \quad$ Guardar en EndNote online
\end{tabular}

Fuente: captura de pantalla de WoS.

b) Análisis de documentos.

La página de análisis de WoS, a la que accedemos desde la opción Analizar resultados, presenta un diseño de la interfaz altamente funcional.

En la izquierda, tenemos una lista de las facetas o parámetros de análisis, y en el cuerpo central los datos correspondientes a la primera de ellas. En el caso de WoS, la lista de facetas de distribución se amplía con un total de 16, contra las siete de Scopus.

En la imagen 14, vemos la distribución correspondiente a las categorías de Web of Science, mostrada en forma de mapa de árbol, por ser la opción por defecto (la podemos cambiar por un gráfico de barras). 
Imagen 14. La atractiva y funcional página de análisis de resultados de WoS con uno de los mapas de árbol interactivos



Fuente: captura de pantalla de WoS.

Este mapa es interactivo en varios sentidos ya que, además de poder modificar alguna de sus variables -como el tipo de gráfico y el número de elementos- al desplazar el cursor por cada recuadro del mapa, aparece una ficha emergente con información relacionada y un enlace a los registros afectados.

c) Análisis de citas.

Para acceder al análisis de citas disponemos de un enlace en la página de resultados (imagen 13) y también podemos acceder desde la página de distribución de datos. La parte más importante del análisis de citas queda recogido por los cuatro cuadros que vemos a continuación:

Imagen 15. Diferentes componentes del análisis de citas de la página de resultados de WoS

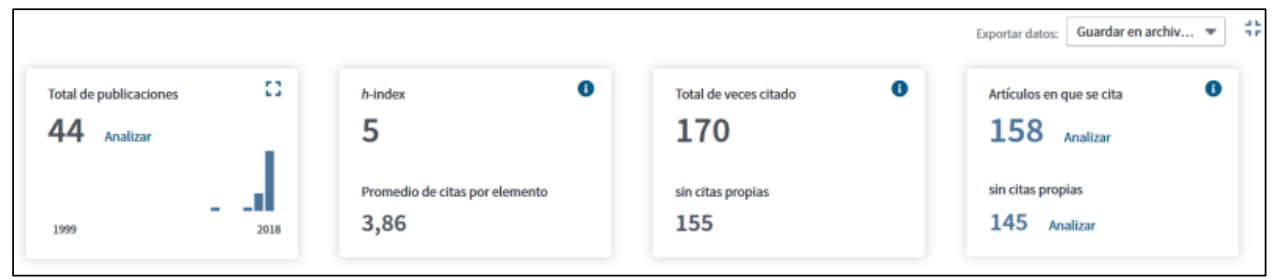

Fuente: captura de pantalla de WoS.

Mediante los cuatro cuadros mostrados en la captura precedente (imagen 15) vemos el número total de publicaciones analizadas y la distribución por 
años — se puede agrandar-, el índice $h$ y el promedio de citas, el número total de citas recibidas - con y sin autocitas-y el número total de artículos en que se produce la cita, con la opción de poder acceder a los mismos.

\subsection{Análisis de fuentes y autores}

Además de los análisis que hemos revisado someramente, ambas bases de datos ofrecen formas de análisis de la información que son independientes de la consulta. Se trata de análisis de fuentes (revistas científicas) y de perfiles de autores, fundamentalmente.

\subsubsection{Análisis de fuentes y autores en Scopus}

En el caso de Scopus, CiteScore y Author Profile son los dos índices, de revistas científicas y de autores respectivamente, que esta importante base de datos ofrece en abierto, por lo cual su consulta es directa y sin barreras.

Esto es, se pueden consultar como parte de la base de datos Scopus, pero también están disponibles en abierto (www.scopus.com) aunque requiere una suscripción gratuita. En lo que sigue, examinamos ambas.

a) CiteScore: índice y métricas de revistas.

CiteScore es la denominación de la métrica que utiliza la base de datos Scopus para determinar la influencia de una revista. El cálculo tiene una base conceptual similar al Journal Impact Factor (JIF) que utiliza Web of Science, pero con dos diferencias, cuyas consecuencias son muy importantes.

En primer lugar, utiliza una ventana de citación de cuatro años, mientras que JIF, utiliza dos años. En segundo lugar, CiteScore incluye todas las revistas de todas las disciplinas de la base de datos Scopus (imagen 16), mientras que JIF deja fuera a las humanidades.

Hay otras diferencias, referidas a la simetría - misma fuente de datospara el numerador y el denominador en el cálculo del factor de impacto, que tiene también como consecuencia una mayor transparencia a favor de CiteScore, lo que permite su reproducibilidad, cosa que se considera no garantizada en el caso del JIF.

Una de las formas más útiles de utilización de CiteScore para los investigadores puede ser consultar listas de revistas de un área determinada. 
Imagen 16. Opciones del índice CiteScore de Scopus

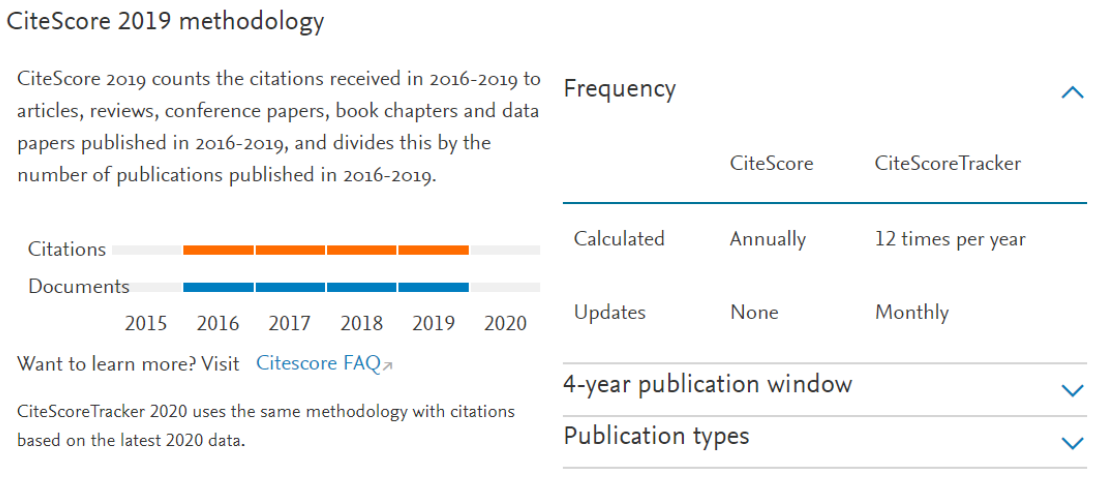

\begin{tabular}{lll} 
& CiteScore & CiteScoreTracker \\
\hline Calculated & Annually & 12 times per year \\
Updates $\quad$ None & Monthly & \\
\hline 4-year publication window & \\
\hline Publication types & \\
\hline
\end{tabular}

Fuente: captura de pantalla de CiteScore.

Como vemos en la imagen 17, en que tomamos como ejemplo a la comunicación, este listado muestra el número total (310 en este caso). Después, tenemos la lista de las revistas, que podemos ordenar por título o por cualquiera de las otras seis columnas entre las que figura el índice CiteScore. Así como tenemos un enlace que nos permite descargar la lista completa de revistas Scopus en una hoja de cálculo.

Imagen 17. Listado de las revistas del área comunicación en CiteScore de Scopus

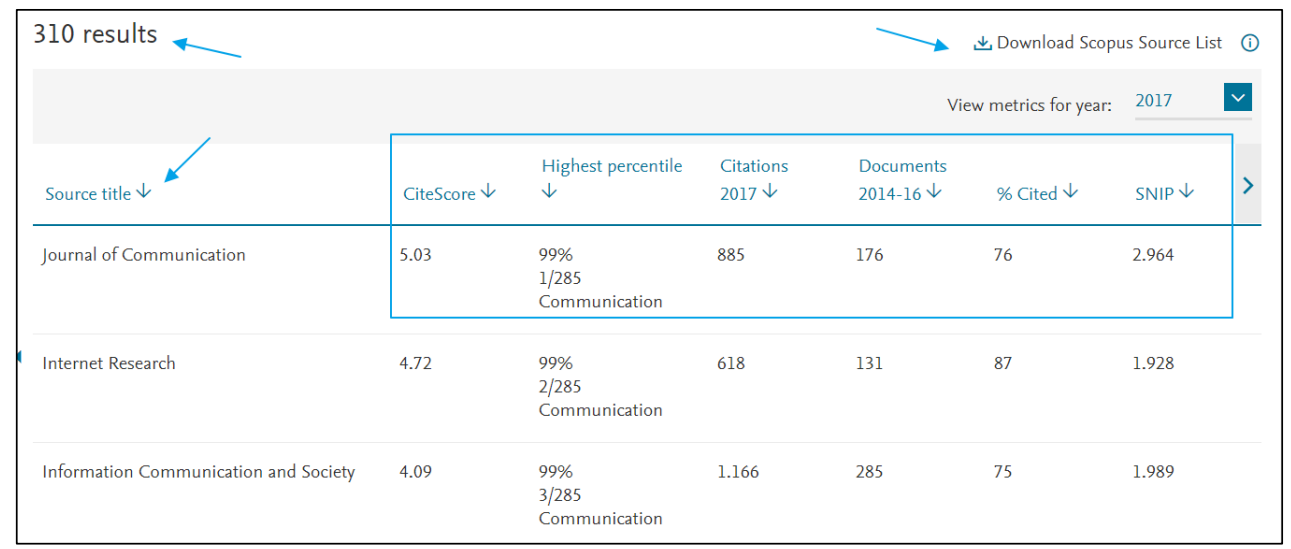

Fuente: captura de pantalla de CiteScore.

A partir de esta lista, podemos recorrer con comodidad la totalidad de los títulos que la componen y, si hacemos clic en cualquiera de ellos, tenemos la completísima ficha de información de cada revista que hemos podido ver en 
capturas precedentes, lo que incluye además un enlace a la página web de la editorial de la misma.

b) Author Profiles.

Como ya hemos señalado, Author Profiles, por su parte, consiste en proporcionar información sobre autores, tal como podemos ver en el siguiente ejemplo (imagen 18).

Imagen 18. Ficha de un autor en Author Profiles de Scopus

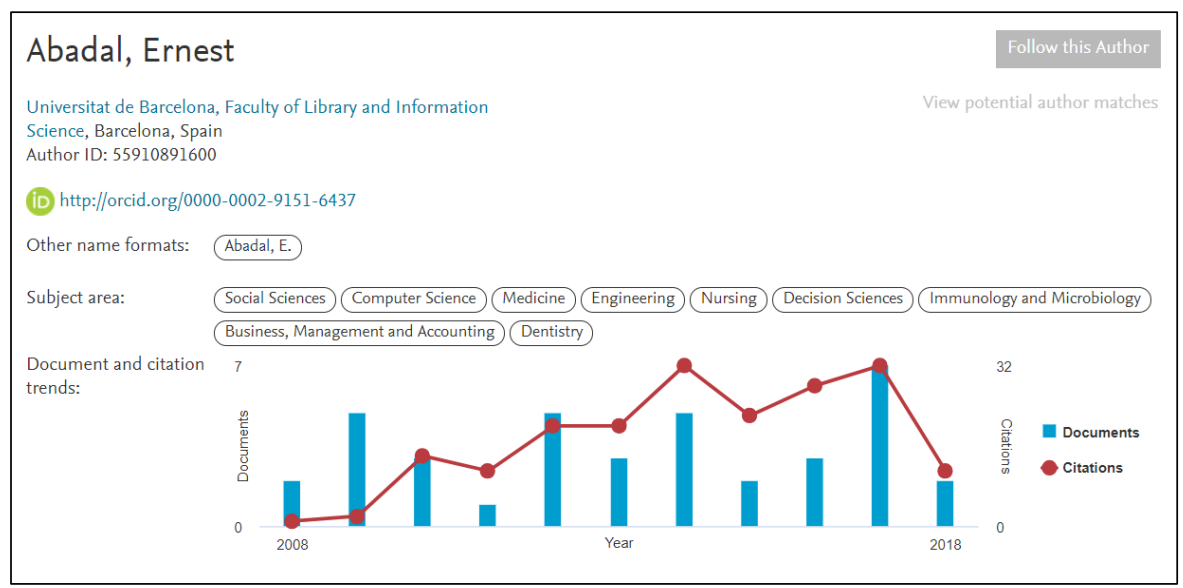

Fuente: captura de pantalla de Author Profiles.

Podemos ver una información muy detallada sobre el autor que, por cierto, toma por defecto los últimos 10 años. Cabe señalar que, además de los numerosos enlaces que presenta, el gráfico es interactivo: al situar el cursor sobre cada barra aparece información detallada del año en cuestión.

\subsubsection{Análisis de fuentes y autores en Web of Science}

En el caso de WoS, InCites es la aplicación independiente de Web of Science (WoS) para llevar a cabo análisis de la producción científica que no están vinculados con resultados de búsquedas. Dicho de otro modo, InCites proporciona métricas que se refieren a la totalidad de las informaciones disponibles en las bases de datos de Web of Science.

InCites es, por tanto, un sistema de información para conocer con un estimable grado de aproximación el estado de la investigación en general, ya sea en todo el mundo, en una región, en una disciplina o con relación a una institución o, incluso, con relación a un autor. 
Esta aplicación incluye la consulta de los famosos Journal Citation Reports (JCR) en la que se basan los estudios de impacto de Web of Science, y que utilizan agencias y universidades como criterio para la evaluación de investigadores o de departamentos de universidad muchas veces de forma única, desplazando la posibilidad de utilizar otros indicadores, como el mencionado CiteScore.

En todo caso, WoS proporciona dos productos principales denominados, respectivamente: Journal Impact Factor (JIF), como parte de los Journal Citation Reports (JCR); y Essential Indicators, que es un conjunto de datos globales o desagregados de la ciencia.

a) JIF en Journal Citation Reports (JCR).

Como ya hemos señalado, Journal Citation Reports es una de las dos herramientas disponibles en InCites. Es, con diferencia, la más conocida ya que incluye, entre otras métricas, el factor de impacto de las publicaciones de Web of Science.

Su importancia es decisiva en ámbitos de las ciencias médicas, experimentales y tecnológicas, pero su utilidad queda muy limitada en otros ámbitos, dado que excluye expresamente a las revistas de Humanidades.

Como se observa en la imagen 19, podemos examinar el JIF dentro de los JCR de las publicaciones de un sector académico mediante el uso de la opción Select Categories. En este caso, hemos elegido ver las revistas de comunicación.

Imagen 19. Journal Citation Reports (JCR) de las revistas de comunicación



Fuente: captura de pantalla de InCites de WoS. 
Los JCR nos permiten conocer las métricas de las revistas presentes en WoS de un país determinado, como podría ser el ejemplo España. En el caso de comunicación, aunque sin duda los JCR pueden resultar de utilidad, tenemos el importante lastre señalado de que no incluye áreas académico-científicas completas, al dejar fuera a las revistas de artes y humanidades.

b) Essential Science Indicators.

Es la segunda de las herramientas de InCites y, como indica su nombre, proporciona una serie de indicadores esenciales de la producción de la ciencia en todo el mundo.

Imagen 20. Mapa interactivo en Essential Science Indicators

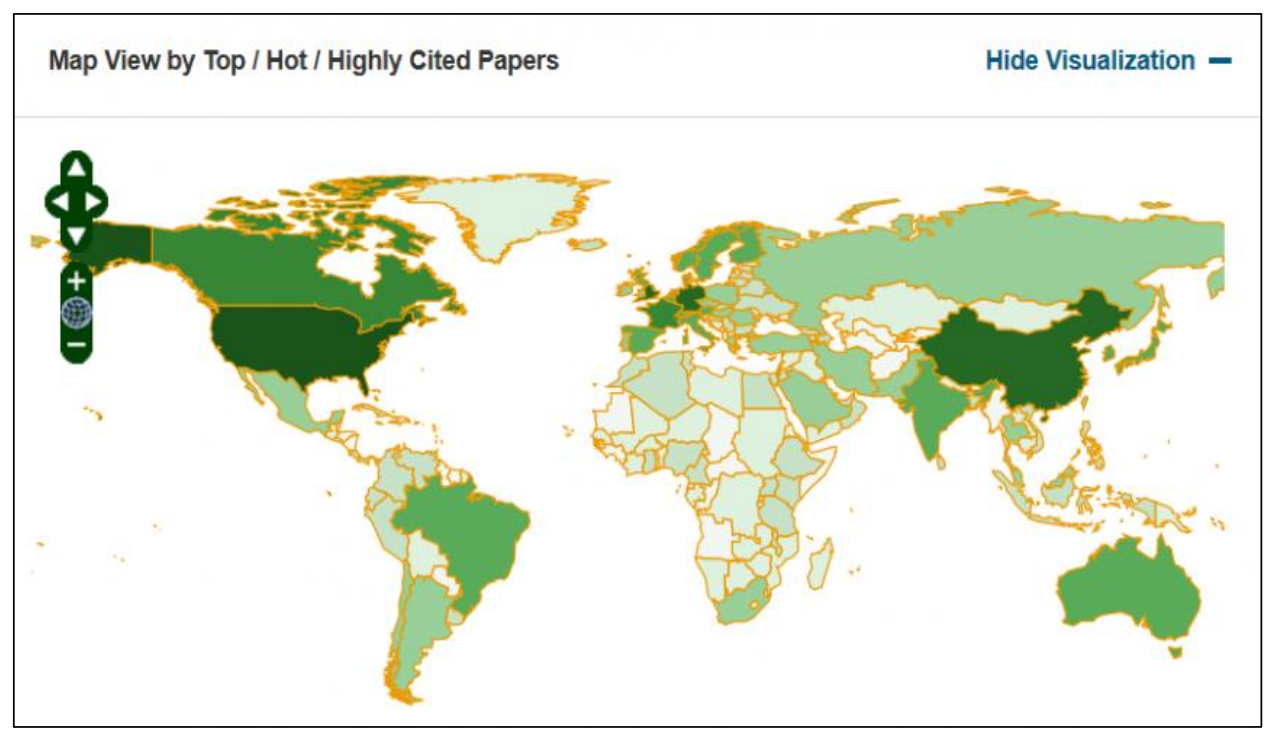

Fuente: captura de pantalla de InCites de WoS.

Tal como se aprecia en la imagen 20, en la parte superior de la página principal de Essential Science Indicators de InCites se muestra un mapa interactivo en el que podemos seleccionar el país del que queremos información.

Finalmente, otra visualización de información de gran utilidad en InCites es la lista de las revistas con mayor factor de impacto de todo el mundo (imagen 21), la cual incluye indicaciones de diversas métricas. 
Imagen 21. Lista mundial de revistas con mayor factor de impacto

\begin{tabular}{|c|c|c|c|c|c|}
\hline \multicolumn{2}{|c|}{ Report View by Selection } & \multirow[b]{2}{*}{$\begin{array}{l}\text { Web of Science } \\
\text { Documents }\end{array}$} & \multirow[b]{2}{*}{ Cites - } & \multirow[b]{2}{*}{ Cites/Paper } & Customize \\
\hline $\begin{array}{l}\text { Total: } \\
22\end{array}$ & Research Fields & & & & Top Papers \\
\hline 1 & $\begin{array}{l}\text { CLINICAL } \\
\text { MEDICINE }\end{array}$ & $2,484,987$ & $30,018,036$ & 12.08 & $24.67^{\circ}$ \\
\hline 2 & CHEMISTRY & $1,569,274$ & $21,097,108$ & 13.44 & 15,61 \\
\hline 3 & PHYSICS & $1,048,823$ & $11,075,207$ & 10.56 & $\square$ \\
\hline 4 & $\begin{array}{l}\text { BIOLOGY \& } \\
\text { BIOCHEMISTRY }\end{array}$ & 675,187 & $10,575,992$ & 15.66 & $\square$ \\
\hline 5 & $\begin{array}{l}\text { MOLECULAR } \\
\text { BIOLOGY \& } \\
\text { GENETICS }\end{array}$ & 423,824 & $9,830,547$ & 23.19 & 4,17 \\
\hline
\end{tabular}

Fuente: captura de pantalla de InCites de WoS.

\section{Discusión}

Dadas las características de las bases de datos examinadas, lo óptimo es combinar o utilizar ambas para investigar en comunicación (por ejemplo, para llevar a cabo trabajos de revisión) o en procesos de evaluación de la ciencia.

Por otro lado, es frecuente observar procesos de revisión así como de evaluación de la ciencia que se limitan a Web of Science o que marginan a Scopus. Sin embargo, como resultado de estos análisis, podemos señalar que, en caso de tener que elegir entre una u otra base de datos, la elección lógica debería recaer en Scopus, ya que como vemos es, con diferencia, la que contiene un mayor número de publicaciones no solamente en ciencias sociales y humanidades, sino como consecuencia lógica, en comunicación.

Insistimos que, mientras que siempre será óptimo contar con las dos, no hay motivo para excluir a Scopus si por alguna razón debemos usar una sola de estas bases de datos. Dicho aún de otro modo, mientras que no requiere mayor justificación usar solamente Scopus, en el caso de comunicación, habría que justificar muy bien usar solamente WoS.

Este uso combinado preferente se refiere a procesos típicos de obtención de información, como por ejemplo, para llevar a cabo una revisión bibliográfica, ya sea de tipo sistematizada o no. También, para identificar corrientes temáticas, publicaciones, centros de investigación, entre otros.

En otros procesos, como los que forman parte de los de evaluación frente a departamento de universidad o agencias de evaluación, a riesgo de ser reiterativos, vuelve a ser oportuna la consideración de que lo óptimo es usar ambas, pero si por alguna razón hubiera que usar solamente una la que menos justificado resulta discriminar es Scopus. 


\section{Conclusiones}

Hemos visto que la principal diferencia de Scopus y Web of Science se refiere a la cobertura relativa, derivada a su vez de la superior cobertura de Scopus de publicaciones de ciencias sociales y humanidades, lo que se puede considerar un mayor yacimiento de información para investigadores en comunicación, a salvo de proyectos muy determinados donde puede haber variaciones en esta consideración de validez general y, por tanto, no necesariamente particular.

Esta mayor cobertura a favor de Scopus tiene diferentes dimensiones. Por un lado, es evidente que proporciona una base de datos más amplia donde llevar a cabo las búsquedas para fundamentar investigaciones.

Pero otro aspecto muy importante se refiere a los análisis de la información. Scopus utiliza un solo índice unificado, donde todas las revistas que integran su base de datos forman parte del índice, sin distinción de áreas, aunque también dispone de índices ponderados que ajustan las métricas a los hábitos de citación de cada gran área: humanidades, ciencias sociales, ciencias experimentales y tecnológicas.

En cambio, Web of Science, en sus Journal Citations Reports (JCR), solamente incluye dos de sus ocho índices, esto es, solamente incluye las revistas de ciencias y las de ciencias sociales. Las revistas de humanidades quedan fuera de los JCR. Por lo tanto, su índice no solamente es mucho más limitado en la base que utiliza — se estima que Scopus incluye en total unas 12.000 revistas más que $\mathrm{WoS}_{-}$, sino que, como señalamos, revistas de áreas completas, como historia, quedan fuera de esta clase de índices que son independientes de la consulta.

Recordemos que, en cambio, en los análisis relacionados con una búsqueda, ni la base de datos Scopus ni la base de datos WoS presentan diferencias o deficiencias remarcables. Por tanto, en este aspecto son equiparables entre ellas y ambas ofrecen muy buenos recursos a los investigadores.

Lo ideal, qué duda cabe, para un equipo de investigadores es poder utilizar ambas bases de datos, ya que pese a la mayor cobertura de Scopus, como ya hemos señalado, no se trata de un superconjunto de WoS, sino que hay publicaciones presentes en WoS que no lo están en Scopus y viceversa.

Como cierre de estas conclusiones, el diagnóstico más compacto que podemos ofrecer es el siguiente: si por alguna razón un equipo de investigadores en comunicación social tuviera que elegir una de las dos bases de datos, la racionalidad está a favor de Scopus. Los motivos son tres: primero, por su superior cobertura de publicaciones en el ámbito de las ciencias sociales y las humanidades. Segundo, por utilizar índices unificados en los que no se discriminan a las 
humanidades. En tercer lugar, por presentar políticas unificadas de selección de revistas científicas que se aplican a todas las disciplinas.

En cambio, WoS, que sin duda es una excelente base de datos para investigadores en ciencias experimentales y tecnológicas, pero no así para los de las humanidades. Su política de dejar fuera de sus JCR a ramas enteras de la ciencia perjudica notablemente esta base de datos en el hipotético caso, que hemos mencionado, de tener que elegir una u otra.

La situación cambia si los criterios de uso no son los que se refieren a cobertura y a sus índices y métricas de revistas y perfiles de autores, sino a criterios de agencias de evaluación que, por un reflejo derivado de las ciencias experimentales y tecnológicas, siguen dando una cierta prioridad a WoS. En este caso, el análisis precedente retrocede ante lo que podemos considerar prejuicios que se aplican sin más por falta de reflexión, como mucho tememos.

\section{Referencias bibliográficas}

CHUNG, C.J., \& PARK, H.W. (2012). Web visibility of scholars in media and communication journals. Scientometrics, 93(1), 207-215. https://doi.org/10.1007/s11192-012-0707-8

CoDinA, L. (2011). Sistemas de búsqueda y obtención de información. In F. Cacheda-Seijo, J.M. Fernández-Luna, \& J.-F. Huete-Guadix (Eds.), Recuperación de información: un enfoque práctico y multidisciplinar (pp. 191-232). Ra-Ma. https://bit.ly/36c9L6r

CoDinA, L. (2017). Investigación con bases de datos. Estructura y funciones de las bases de datos académicas. Análisis de componentes y estudio de caso. https:// repositori.upf.edu/handle/10230/28135

CoDinA, L. (2018). Sistemas de búsqueda y obtención de información: componentes y evolución. Anuario ThinkEPI, 12, 77. https://doi.org/10.3145/thinkepi.2018.06

COSTA-SÁNCHEZ, C. (2017). Análisis de la productividad y visibilidad en Scopus de los investigadores españoles en Comunicación. Observatorio $\left(O B S^{*}\right)$, 11(3), 1-16. https://doi.org/10.15847/obsOBS11320171030

DE-FILIPPO, D. (2013). Spanish scientific output in communication sciences in WOS. The scientific journals in SSCI (2007-12). Comunicar, 21(41), 25-34. https://doi.org/10.3916/C41-2013-02

DELGADO-LóPEZ-CóZAR, E., \& REPISO-CABALLERO, R. (2013). The impact of scientific journals of communication: Comparing Google Scholar Metrics, Web of Science and Scopus. Comunicar, 21(41), 45-52. https: //doi.org/10.3916/C41-2013-04

DEmeTER, M. (2018a). Changing Center and Stagnant Periphery in Communication and Media Studies: National Diversity of Major 
International Journals in the Field of Communication from 2013 to 2017. International Journal of Communication, 12(0). https://ijoc.org/index.php/ijoc/article/view/9122

DEMETER, M. (2018b). Nobody Notices It? Qualitative Inequalities of Leading Publications in Communication and Media Research. International Journal of Communication, 12(0). https: //doi.org/1932-8036/20180005

GASPARYAN, A.Y. (2013). Bibliographic Databases: Some Critical Points. Journal of Korean Medical Science, 28(6), 799.

https://doi.org/10.3346/jkms.2013.28.6.799

Kousha, K., ThelWALL, M., \& REZAiE, S. (2010). Using the Web for research evaluation: The Integrated Online Impact indicator. Journal of Informetrics, 4(1), 124-135. https://doi.org/10.1016/j.joi.2009.10.003

LEYDESDORFF, L., DE MOYA-ANEGón, F., \& DE NoOY, W. (2016). Aggregated journal-journal citation relations in Scopus and Web of Science matched and compared in terms of networks, maps, and interactive overlays. Journal of the Association for Information Science and Technology, 67(9), 2194-2211. https://doi.org/10.1002/asi.23372

Montero-Díaz, J., Cobo, M.J., GuTiÉrRez-SALCEdo, M., SEGAdo-BoJ, F., \& HerRerAVIEDMA, E. (2018). A science mapping analysis of 'Communication' WoS subject category (1980-2013). Comunicar, 26(55), 81-91. https: //doi.org/10.3916/C55-2018-08

Morales-VARGAS, A., PEDRAZA-JIMÉnEZ, R., \& Codina, L. (2020). Website quality: An analysis of scientific production. Profesional de La Información, 29(5), e290508. https://doi.org/10.3145/epi.2020.sep.08

Rogel-SAlaZAR, R., SANTIAGo-BAuTISTA, I., \& MARTínEZ-DomínGUEZ, N. (2017). Revistas científicas latinoamericanas de Comunicación indizadas en WoS, Scopus y bases de datos de Acceso Abierto. Comunicación y Sociedad, 30, 167-196. https://doi.org/10.32870/cys.v0i30.6514

TÚÑEZ-LóPEZ, M. (2013). El 'índice h' de la investigación en Comunicación en España, Portugal y Latinoamérica: Web of Knowledge (WoK), Scopusy Google Scholar Metrics. Communication and Society, 26(4), 53-75. https://doi.org/10.15581/003.26.4.53-75

WEINHOLD, T., OETTL, S., \& BEKAVAC, B. (2011). Heuristics for the Evaluation of Library Online Catalogues. In C. Katsirikou, A and Skiadas (Ed.), New Trends in Qualitative and Quantitative Methods in Libraries (pp. 425-432). World Scientific. https://doi.org/10.1142/9789814350303_0052 